\title{
The First Asymmetry Measurements in High-Energy Polarized Proton-Nucleus Collision at PHENIX-RHIC
}

\author{
Itaru Nakagawa ${ }^{1,2, a}$ for the PHENIX Collaboration \\ ${ }^{1}$ RIKEN, 2-1 Hirosawa, Wako, Saitama, 351-0198, Japan \\ ${ }^{2}$ Riken-BNL Research Center, 20 Pennsylvania Avenue BIdg.510A, Upton, NY 11973, U.S.A.
}

\begin{abstract}
The single spin asymmetries in very forward neutron production had been first observed about a decade ago at RHIC in transversely polarized proton + proton collision at $\sqrt{s}=200 \mathrm{GeV}$. Although neutron production near zero degrees is well described by the one-pion exchange (OPE) framework, the OPE appeared to be not satisfactory to describe the observed analyzing power $A_{\mathrm{N}}$. The absorptive correction to the OPE generates the asymmetry as a consequence of a phase shift between the spin flip and non-spin flip amplitudes. However the amplitude predicted by the OPE is too small to explain the large observed asymmetries. Only the model which introduces interference between major pion and small $a_{1}$-Reggeon exchange amplitudes has been successful in reproducing the experimental data. During RHIC Run-15, RHIC delivered polarized proton collisions with $\mathrm{Au}$ and $\mathrm{Al}$ for the first time, enabling the exploration of the mechanism of transverse single-spin asymmetries with nuclear collisions. A very striking A-dependence was discovered in very forward neutron production at PHENIX in transversely polarized proton + nucleus collision at $\sqrt{s}=200 \mathrm{GeV}$. Such a dependence has not been predicted from the existing framework which has been succesful in proton + proton collision. In this report, experimental and theoretical efforts are discussed to disentangle the mysterious A-dependence in the very forward neutron asymmetry.
\end{abstract}

\section{Introduction}

The single spin asymmetry of very forward (almost zero degree) was first discovered[1] at RHIC in transversely polarized proton+proton collision at $\sqrt{s}=200 \mathrm{GeV}$. Neutrons were measured by a zero-degree calorimeter (ZDC) [2] with a position-sensitive shower-maximum detector (SMD).

The magnitude of observed asymmetry of the neutron which has the energy fraction of incident proton $x_{\mathrm{F}}=E_{\mathrm{n}} / E_{\mathrm{p}} \geq 0.4$ was several percent. This asymmetry was unexpectedly large. The production mechanism of the such a neutron is driven by soft process and of which cross section is well described by one pion exchange (OPE) model. However the predicted asymmetry within the OPE framework appeared to be very tiny and far underestimated the data[3]. The forward neutron asymmetry is formulated as

$$
A_{\mathrm{N}} \propto \phi_{\text {flip }} \phi_{\text {non-flip }} \sin \delta
$$

\footnotetext{
ae-mail: itaru@ riken.jp
} 
where $\phi_{\text {flip }}\left(\phi_{\text {non-flip }}\right)$ is spin flip (non-flip) amplitude between incident proton and out-going neutron, and $\delta$ is the relative phase between these two amplitudes. Although the OPE can contribute to both spin flip and non-flip amplitudes, resulting $A_{\mathrm{N}}$ is small due to the small relative phase. The several percent amplitude was generated only by introducing the interference between spin flip $\pi$ exchange and spin non-flip $a_{1}$-Reggeon exchange which has large phase shift in between[3]. Shown in Fig. 1 are observed forward neutron asymmetries in three different collision energies 64,200 , and $500 \mathrm{GeV}$ and plotted as a function of transverse momentum $p_{\mathrm{T}}$ of detected neutron. The data demonstrate the absolute amplitude of the asymmetries grow as a function of $p_{\mathrm{T}}$. These data are well reproduced by the model calculations[3].

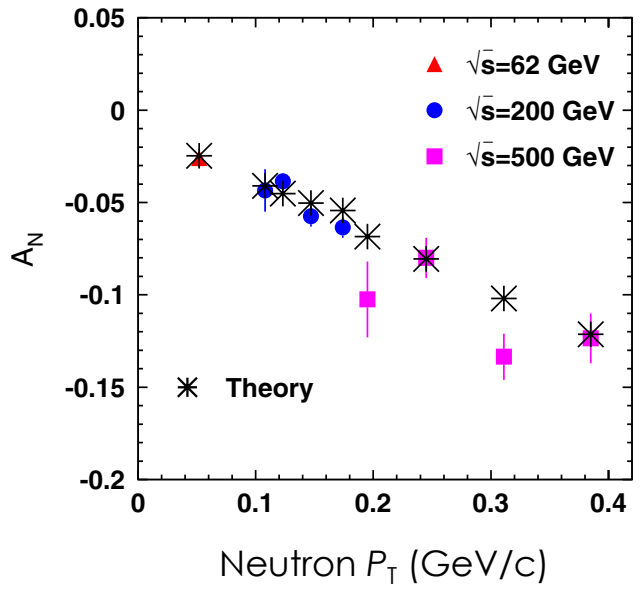

Figure 1. The forward neutron asymmetries observed $\mathrm{p}+\mathrm{p}$ collision system at three different collision energies 64,200 , and $500 \mathrm{GeV}$ plotted as a function of transverse momentum $p_{\mathrm{T}}$. The data points are well reproduced by the model calculations assumes the interference between $\pi$ and $a_{1}$-Reggeon[3].

\section{Experimental Setup}

The ZDC is composed of $\mathrm{Cu}-\mathrm{W}$ alloy absorbers with PMMA-based communication grade optical fibers and it corresponds to 1.7 nuclear interaction length for one module. A photomultiplier collects Cherenkov lights via the optical fibers in one module. As shown in Fig. 2, three ZDCs are located in series at $\pm 18 \mathrm{~m}$ away from the collision point within the small acceptance, covering $10 \mathrm{~cm}$ in the transverse plane. It has 5.1 nuclear interaction length and 149 radiation length by combining three ZDC modules, and has energy resolution about $20 \%$ for $100 \mathrm{GeV}$ neutron.

The SMD consists of $x-y$ scintillator strip hodoscopes and they are inserted between the first and second ZDC modules at the position of maximum hadronic shower approximately. The $x$-coordinate (horizontal) is sampled by 7 scintillator strips of $15 \mathrm{~mm}$ width, while the $y$-coordinate (vertical) is sampled by 8 strips of $20 \mathrm{~mm}$ width, tilted by 45 degree. It gives a position by calculating the center of gravity of shower generated in the first ZDC module. Its position resolution is estimated to be about $1 \mathrm{~cm}$ for $50 \mathrm{GeV}$ neutron in the simulation study.

The data was collected by triggers employing the ZDCs and Beam Beam Counters (BBCs). One $\mathrm{BBC}$ consists of 64 sets of PMTs and $3 \mathrm{~cm}$ thick quartz Cherenkov radiator. BBCs are mounted 


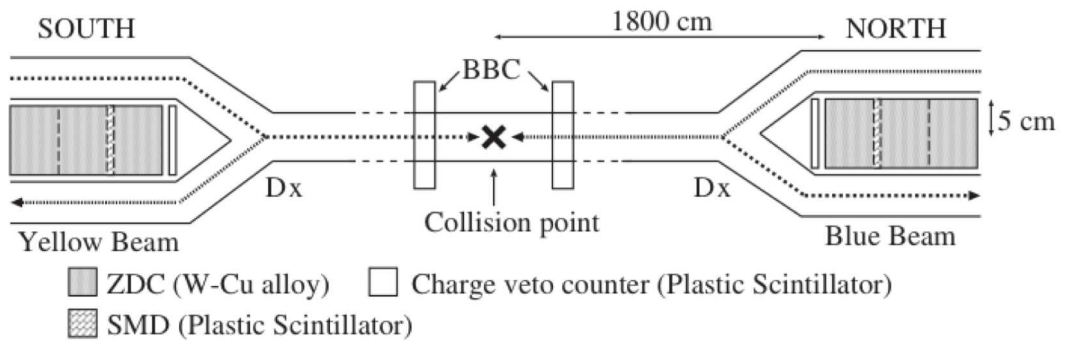

Figure 2. The experimental setup for ZDC and BBC detectors. The ZDCs were located $\pm 18 \mathrm{~m}$ downstream from the collision point. The BBCs were installed around the beam pipe at $\pm 18 \mathrm{~m}$ downstream of the collision point.

around beam pipe located $\pm 144 \mathrm{~cm}$ away from the collision point. They cover $\pm(3.0-3.9)$ and $2 \pi$ in $\eta$ and $\phi$ spaces, respectively.

\section{New Forward Neutron Asymmetries in $\mathrm{p}+\mathrm{A}$}

In year 2015, the first attempt was made to collide the transversely polarized proton and nucleus in RHIC at $\sqrt{s}=200 \mathrm{GeV}$. In order to explore the atomic mass number (A) evolution of the asymmetry, the forward ( $\mathrm{p}$-going side) neutron asymmetries were measured for $\mathrm{p}+\mathrm{Au}$ and $\mathrm{p}+\mathrm{Al}$ collisions. Since the proton beam is unpolarized at LHC, the measurement is the high energy frontier of the polarized proton and nucleus collision. The existing polarized proton+nucleus experiments are carried out in one order of magnitude lower $\sqrt{s}$, for instance, at RHIC[5] and Fermilab [11] using polarized proton beam and fixed nuclear targets.

The asymmetry analysis basically followed the same procedures and conditions as previous $\mathrm{p}+\mathrm{p}$ measurements. The measurement in $\mathrm{p}+\mathrm{p}$ collision system was repeated in year 2015 and resulting asymmetry was consistent with previous measurements. The ZDC acceptance was segmented into eight azimuthal bins and evaluated left-right raw asymmetries $\epsilon_{N}^{r a w}$ defined by the Eqn. (2) in the each bin independently.

$$
\epsilon_{N}^{r a w}(\phi)=\frac{N^{+}(\phi)-N^{-}(\phi)}{N^{+}(\phi)+N^{-}(\phi)}
$$

where $N^{+}(\phi)$ and $N^{-}(\phi)$ are number of neutrons observed in the given azimuthal $\phi$ bin when protons are polarized up and down, respectively.

The observed $\phi$ dependent asymmetries normalized by the proton polarization $P$ are plotted in Fig. 4 as a function of $\phi$. The $\phi=0$ is set by the polarization direction of the proton. The analyzing power $A_{\mathrm{N}}$ is evaluated by fitting the data with a sine function:

$$
\frac{\epsilon_{N}^{r a w}(\phi)}{P}=A_{\mathrm{N}} \sin \left(\phi-\phi_{0}\right)
$$

where $\phi_{0}$ allows a deviation of the maximum asymmetry axis from vertical.

Fig.4 shows preliminary results of forward neutron $A_{\mathrm{N}}$ measurements in 2015 . The red points are the results of forward neutron $A_{\mathrm{N}}$ inclusive measurements. Surprisingly, they show unexpectedly strong mass number (A) dependence. The asymmetry even flips the sign from $\mathrm{p}+\mathrm{p}, \mathrm{p}+\mathrm{Al}$ to $\mathrm{p}+\mathrm{Au}$. 


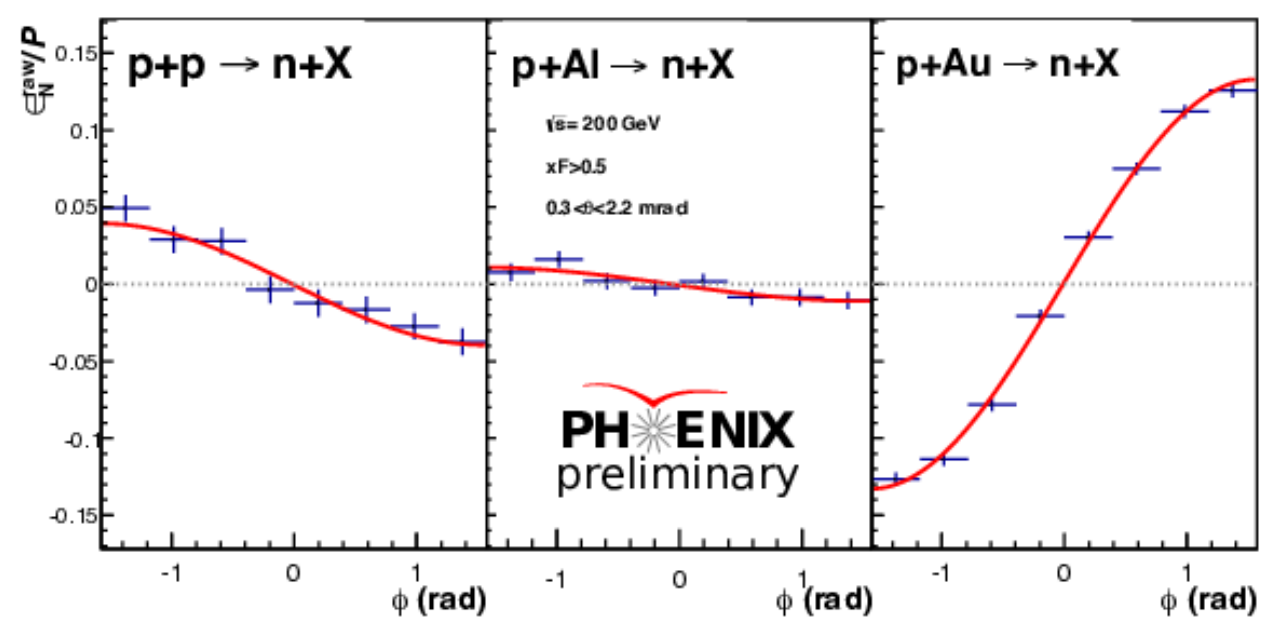

Figure 3. (Observed azimuthal angular modulations of the inclusive forward neutron production in $p+p$ (left), $\mathrm{p}+\mathrm{Al}$ (middle) and $\mathrm{p}+\mathrm{Au}$ (right) collisions in year 2015 .

The $\mathrm{p}+\mathrm{Au}$ data point shows magnificently large $A_{\mathrm{N}}$ of about 0.18 which is three times larger than that of $\mathrm{p}+\mathrm{p}$ in absolute amplitude. The existing $\pi$ and $a_{1}$-Reggeon interference framework predicts only moderate evolution as growing $\mathrm{A}$ and does not have any mechanism to flip the sign of $A_{\mathrm{N}}$ in any $\mathrm{p}+\mathrm{A}$ collision systems.

More interestingly, another drastic dependence of $A_{\mathrm{N}}$ was observed in correlation measurements in addition to the inclusive neutron. In these measurements, another out-going charged particle was either tagged or vetoed within the acceptance of the BBC in both North and South arms. The BBCs cover such a limited acceptance as described in Section 2, but the resulting asymmetries behaved remarkably contradicts. Once BBC hits (BBC tagging) are required in both arms (green data points), the drastic behavior of inclusive $A_{\mathrm{N}}$ is vanished and no flipping sign was observed between $\mathrm{p}+\mathrm{p}$ and $\mathrm{p}+\mathrm{Au}$. On the contrary, the asymmetries are pushed even more positive for $\mathrm{p}+\mathrm{Al}$ and $\mathrm{p}+\mathrm{Au}$ data points once no hits in $\mathrm{BBC}$ are required (BBC vetoed) as represented by blue data points.

\section{Ultra-Peripheral Collision (Primakoff) Effects}

The electro-magnetic (EM) interaction was ignored in the forward neutron production in the case of $p+p$ collision system. However EM interaction may not be ignorable for $p+A u$ case. In fact the importance of EM interaction was pointed out in high energy forward hadron production [11] and elastic p+A scattering [5] experiments. Due to the smallness of the four momentum transfers of the present kinematics, i.e. $-t \leq 0.5(\mathrm{GeV} / \mathrm{c})^{2}$, the EM interaction may play a role which becomes increasingly important in large atomic number nucleus. The EM field of the nucleus becomes rich source of exchanging photons between the polarized proton. This is known as Primakoff effect or the ultra-peripheral collision (UPC) in heavy ion collider experiments. In the UPC process, there is no charge exchange at the collision vertex unlike $\pi$ or $a_{1}$ meson exchange. The forward neutron in the 


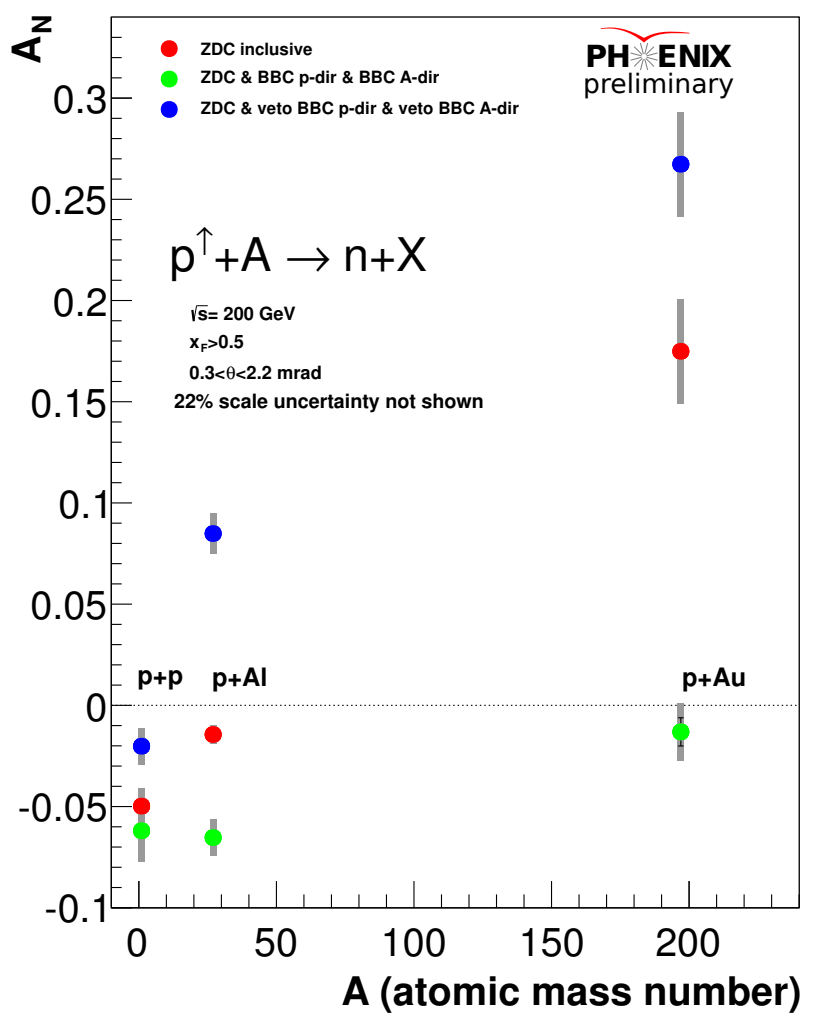

Figure 4. (Observed forward neutron $A_{\mathrm{N}}$ in transversely polarized proton-nucleus collisions. Data points are $\mathrm{A}=1, \mathrm{~A}=27$, and $\mathrm{A}=197$ are results of $\mathrm{p}+\mathrm{p}, \mathrm{p}+\mathrm{Al}$, and $\mathrm{p}+\mathrm{Au}$, respectively. Red, Blue and Green data points are neutron inclusive, neutron $+\mathrm{BBC}$ veto, and $\mathrm{BBC}$ tagged events, respectively

final state then can be produced through the neutron decay channel from the nucleon excitation states as shown in diagrams in Fig. 5.

(a)

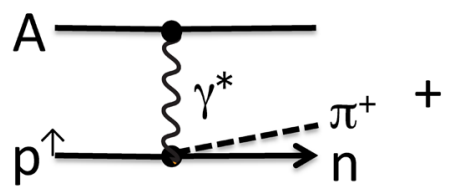

(b)

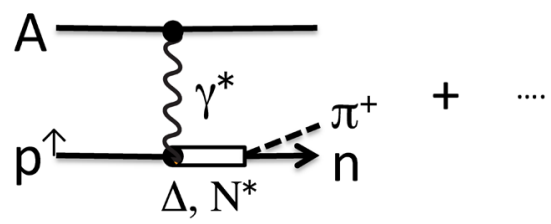

Figure 5. Diagrams of Primakoff process to leave the high- $z$ neutron in the final state to the proton going directions. (a) threshold photo pion production (b) $\Delta$ or $N^{*}$ excitation and its decay into $\mathrm{n}+\pi^{+}$channel. 
The description of $A_{\mathrm{N}}$ is thus extended from Eqn. (1) to Eqn. 5, which includes not only hadronic but also EM amplitudes:

$$
\begin{aligned}
A_{\mathrm{N}} & \propto \phi_{\text {flip }}^{\text {had }} \phi_{\text {non-flip }}^{\text {had }} \sin \delta_{1}+\phi_{\text {flip }}^{\mathrm{EM}} \phi_{\text {non-flip }}^{\text {had }} \sin \delta_{2} \\
& +\phi_{\text {flip }}^{\text {had }} \phi_{\text {non-flip }}^{\mathrm{EM}} \sin \delta_{3}+\phi_{\text {flip }}^{\mathrm{EM}} \phi_{\text {non-flip }}^{\mathrm{EM}} \sin \delta_{4}
\end{aligned}
$$

where 'EM' and 'had' stand for electromagnetic and hadronic interactions, and $\delta_{1} \sim \delta_{4}$ are relative phases, respectively. The second and the third terms are known as Coulomb nuclear interference (CNI) and to be discussed in details in Section 5.

A Monte-Carlo (MC) study was made to predict the kenematic behavior of the process through the Primakoff Effect. The nucleon excitation through Primakoff effect can be quantified into two steps; 1) equivalent photon flux from nuclear Coulomb field, and 2) photo-nucleon excitation. We employed STARLIGHT[6] for modeling nuclear Coulomb field and used Weizsacker-Williams formula[7] to calculate the equivalent photon flux. Among many models are available in the market, we employed SOPHIA model[8] which covers higher excitation states above $\Delta$ region. While the MC is modeling higher excitation states, the first excitation states such as $\Delta$ is rather important in this case because the equivalent photon flux rapidly drops as a function of the photon energy. Threshold pion production even below resonance may play non-negligible role. According to the MC study [9], the neutron and its counter part $\pi^{+}$via Primakoff process is substantially boosted towards the proton beam direction and therefore the fragmenting $\pi^{+}$are mostly emitted in even higher rapidity region than BBC as shown in Fig. 6. Only small fraction $(6 \%)$ of $\pi^{+}$are detected by BBC. Thus EM processes are suppressed in the $\mathrm{BBC}$ tagging events while enhanced in the $\mathrm{BBC}$ vetoed events. As a consequence, one may draw a hypothesis that the moderate $A_{\mathrm{N}}$ evolution of the BBC tagging data are consistent with the prediction based on $\pi$ and $a_{1}$-Reggeon interference scenario because the data are dominated by the hadronic amplitude. Although it is not trivial to predict the sign of the $A_{\mathrm{N}}$ caused by EM amplitude, the observed growing feature of $A_{\mathrm{N}}$ for $\mathrm{BBC}$ vetoed events makes sense if EM amplitude guides to opposite sign of hadronic interactions at least qualitatively. Nevertheless it is not known at all at this moment why EM should have opposite sign nor should have such a large amplitude or large relative phases between hadronic/EM spin-flip and spin-nonflip amplitudes to produce such the large asymmetries. It remains mystery.

\section{Coulomb-Nuclear Interference}

It is known to have finite $A_{\mathrm{N}}$ in the elastic proton + proton (Carbon) scattering process using fixed target with transversely polarized high energy proton beam. In fact the polarization of the proton beams at RHIC are determined by measuring the asymmetry of the elastic scattering on fixed targets. The magnitude of the $\left|A_{\mathrm{N}}\right|$ is $<5 \%$ asymmetry in the momentum transfer region of $0.002<-t<0.012$ in $p+p$, and $p+A$ [5] as shown in Fig. 7 . Because of the smallness of the momentum transfer, the EM interaction plays important role in these processes as shown in the Fig.8 (b), while the hadron interaction amplitude is mainly driven by Pomeron exchange as shown in Fig.8 (a). As expected from the nature of EM interaction, stronger EM field in the large A nucleus results in the distinctive Adependence in the observables. Since this is the elastic scattering, the final states are the same in both EM and hadronic interactions by definition. Thus these two amplitudes interfere and their non-zero relative phase creates finite asymmetry as observed.

The CNI interference in the very forward neutron asymmetry is not trivial as the elastic case since the final states of both EM and hadronic interactions have to exactly match among infinite number 


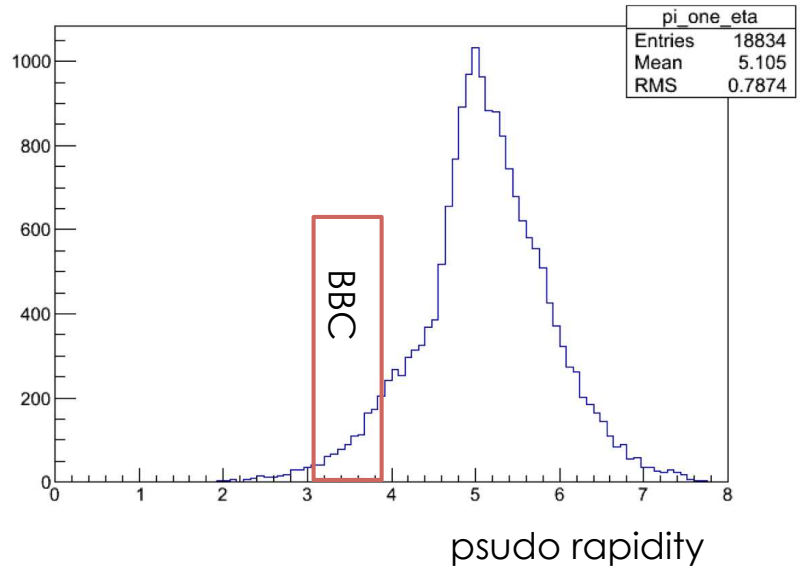

Figure 6. Psudorapidity distribution of $\pi^{+}$production through Primakoff process predicted by MC[9]. The $\pi^{+}$ are mostly emitted to forward psudo rapidity region than BBC covers $3.1<\eta<3.9$.

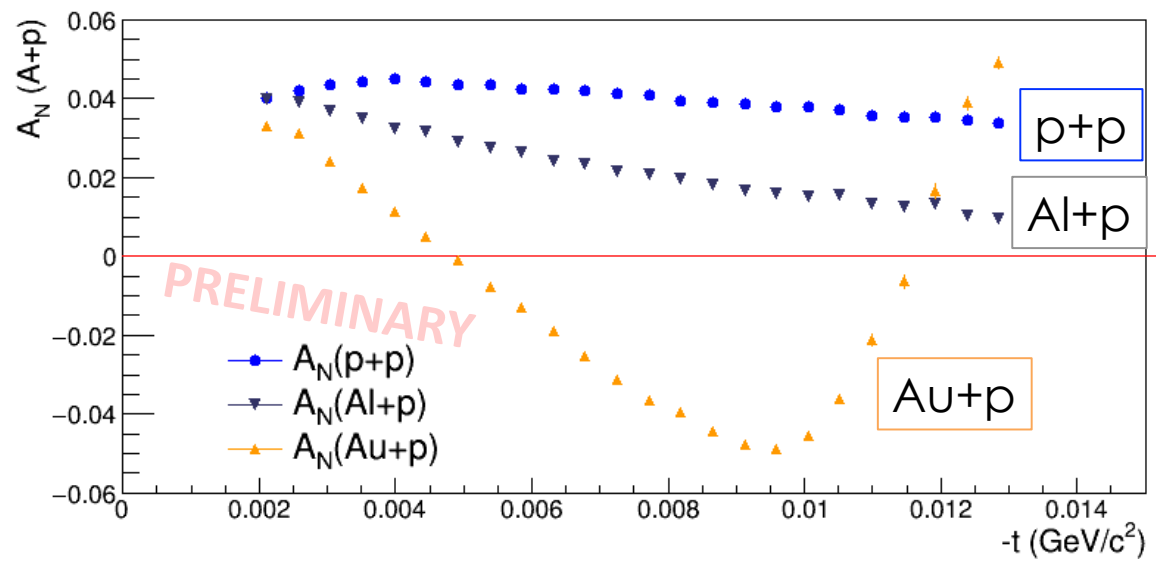

Figure 7. Elastic $A_{\mathrm{N}}$ for $\mathrm{p}+\mathrm{p}, \mathrm{Al}+\mathrm{p}$, and $\mathrm{Au}+\mathrm{p}$ plotted as a function of 4 -momentum transfer $-t$.

of possible multi-particle states. One of the possible leading order diagrams are drawn in Fig.8 (c) for the hadronic and (d) for the EM interactions, respectively. (c) is the inelastic neutron production via Pomeron- $\pi$ scattering, and (d) is $n+\pi^{+}$decay channel of photo- $\Delta$ or $N^{*}$ excitation. Due to the requirement on the same final state, it is natural to consider the lower the multiplicity in the final state, the higher the chance to contribute to CNI. Thus the neutron production via diffractive process is expected to play rather important role CNI terms. On the other hand, the magnitude of $\left|A_{N}\right|$ was only $<5 \%$ level as a consequence of CNI in the even in the $\mathrm{Au}+\mathrm{p}$ elastic case and it is not clear yet if CNI can induce the $\left|A_{N}\right|$ in the order of $25 \%$ for the neutron production. 


\section{Elastic Scattering}

(a)

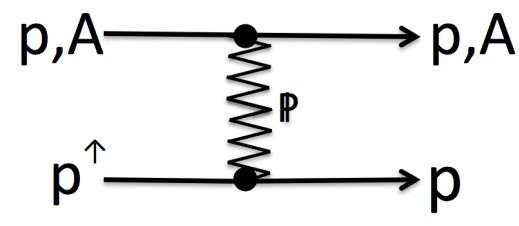

(b)

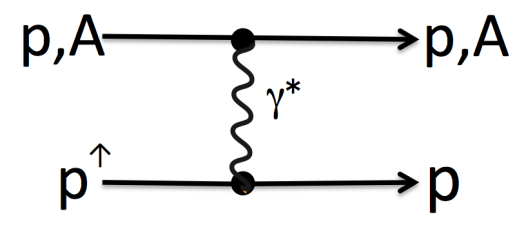

\section{Inelastic Neutron Production}
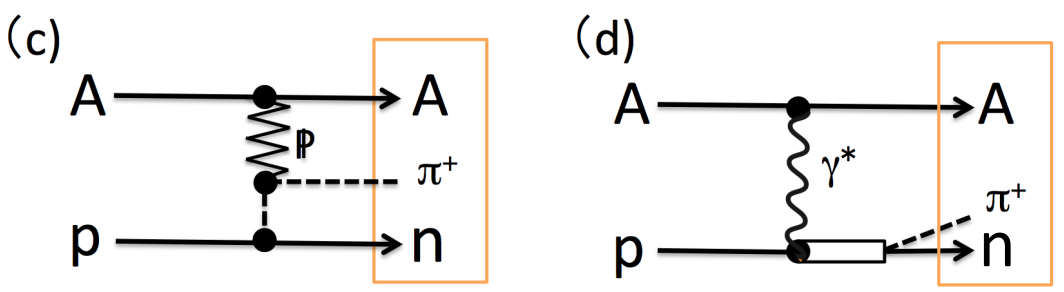

Figure 8. Feynman diagrams of the elastic proton scattering via Pomeron exchange (a) and one-photon exchange (b). The inelastic neutron production via Pomeron- $\pi$ scattering (c), and $\pi+n$ decay channel of photo- $\Delta$ or $N^{*}$ excitation (d).

\section{Fermilab Fixed Target Experiment}

There is one possibly related experimental results were reported by E704 collaboration at Fermilab using a fixed target [11]. They measured $A_{\mathrm{N}}$ in inclusive $\pi_{0}$ production using $185 \mathrm{GeV}$ transversely polarized proton beam on a lead target. The center of mass energy was $\sqrt{s}=19.5 \mathrm{GeV}$, which is one order of magnitude lower than the present experiment. They reported a large $A_{\mathrm{N}}=-0.57 \pm$ 0.12 (statistical) $\pm_{-0.18}^{+0.21}$ (scale uncertainty due to the dilution factor) was observed in $\pi^{0}$ very small $\left|t^{\prime}\right|<0.001(\mathrm{GeV} / \mathrm{c})^{2}$ region $^{1}$ in $p+P b \rightarrow p+\pi^{0}+X$ reaction. Their claim was the cause of the asymmetry comes from the interference between $\Delta$ and $N^{*}$ (Roper) via Primakoff process. Unlike the present experiment, they measured out-going proton in coincidence with pion, they can determine the final state kinematics assuming two body decay of the proton and the lead nucleus remains grand state. The observed $A_{\mathrm{N}}$ neither in the larger $\left|t^{\prime}\right|>0.001(\mathrm{GeV} / \mathrm{c})^{2}$ nor in the invariant mass $M$ region of $\Delta$, i.e. $M<1.36 \mathrm{GeV}$ were zero consistent within their precision. The only invariant mass region $1.36<M<1.52\left(\mathrm{GeV} / \mathrm{c}^{2}\right)$ above $\Delta$ and small $\left|t^{\prime}\right|<0.001(\mathrm{GeV} / \mathrm{c})^{2}$ region showed finite and large asymmetry as described above. They didn't claim the CNI contribution to the finite asymmetry because their $\left|t^{\prime}\right|$ was believed to be sufficiently small. However, the importance of CNI effect was pointed out later by other author [12].

Although the origin of $A_{\mathrm{N}}$ in the Fermilab experiment is still remain unresolved as of today, it may provide some hints to reveal the origin of the present neutron $A_{\mathrm{N}}$. To some extent, the Fermilab and the present experiments are similar if we consider as follows. The Fermilab experiment measures

\footnotetext{
${ }^{1}$ See reference [11] for the definition of $t^{\prime}$.
} 

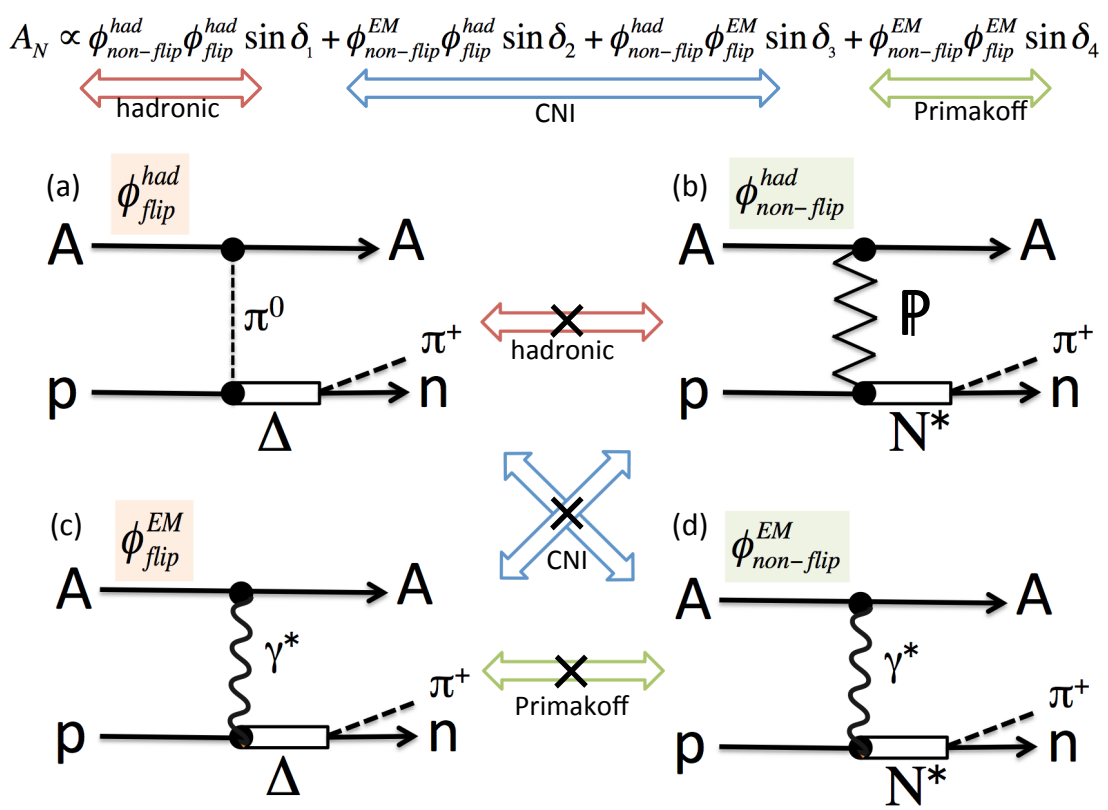

Figure 9. Leading diagrams for the hadronic interaction (top) and EM (bottom) for ( $n+\pi^{+}$) decay channel assuming similar interference effect between $\Delta$ and $N^{*}$ as the Fermilab experiment. (a) spin-flipping $\Delta$ excitation via $\pi^{0}$-exchange (b) spin non-flipping $N^{*}$ excitation via Pomeron exchange, (c) spin-flipping $\Delta$ excitation and (d) spin non-flipping $N^{*}$ excitation via one photon exchange diagrams. The hadronic, CNI, and Primakoff terms of $A_{\mathrm{N}}$ can be given by the interference between spin-flip and non spin-flip amplitudes of hadronic interaction, hadronic and EM interactions, and EM interactions, respectively.

the $\left(\mathrm{p}+\pi^{0}\right)$ decay channel whereas the present experiment measure $\left(\mathrm{n}+\pi^{+}\right)$one. Since their claim was the origin of the asymmetry was the interference between $\Delta$ and $N^{*}$ via Primakoff, the corresponding leading diagrams in the case of hadronic interaction can be one $\pi^{0}$ exchange as shown in Fig. 9(a) for spin flip, and Pomeron exchange as (b) for non-spin flip amplitudes. The diagram (c) and (d) shows the $\left(\mathrm{n}+\pi^{+}\right)$decay channel of the spin flipping photo- $\Delta$ excitation and non spin-flipping photo- $N^{*}$ excitations, respectively. The hadronic, CNI, and Primakoff terms of $A_{\mathrm{N}}$ can be given by the interference between spin-flip and non spin-flip amplitudes of interaction, hadronic and EM interactions, and EM interactions, respectively. These diagrams are not consistent to the leading order diagram raised in Fig. 8(c). In order to be conclusive to judge which diagram is important, quantitative calculation is necessary.

\section{Summary}

We have observed surprisingly strong A-dependence in the forward neutron asymmetry in transversely polarized proton-nucleus collision at $\sqrt{s}=200 \mathrm{GeV}$ in PHENIX using ZDC detector. Such a drastic A-dependence in the forward neutron asymmetry $A_{\mathrm{N}}$ is absolutely unexpected within the current framework although it was successful in reproducing the $A_{\mathrm{N}}$ data in $\mathrm{p}+\mathrm{p}$. Another drastic dependence has been observed in the semi-inclusive measurements by requiring both North and South BBCs 
$3.1<|\eta|<3.9$ to be fired or not-fired in addition to forward neutron. The EM effect may play key role to disentangle these drastic behavior because 1) its amplitude glows as a function of atomic number $\mathrm{Z}$, and 2) the fraction of EM process events are suppressed in BBC fired events while enhanced in BBC vetoed events. 3) Possibly related experiments [5] and [11] points out the importance of EM effects. Further experimental dependency study is certainly be a help to distinguish the hadronic and EM interaction due to their different dependence in impact radius. The corresponding efforts are now underway by segmenting $p_{\mathrm{T}}$ into multiple bins.

\section{References}

[1] Y. Fukao et al., Phys. Lett. B 650, 325 (2007), A. Adare et al.: Phys. Rev. D 88, 032006 (2013).

[2] C. Adler, A. Denisov, E. Garcia, M. J. Murray, H. Strobele, and S. N. White, Nucl. Instrum. Methods Phys. Res., A 470, 488 (2001).

[3] B. Z. Kopeliovich, I. K. Potashnikova, and Ivan Schmidt: Phys. Rev. Lett. 64, 357 (1990).

[4] D. C. Carey et al.: Phys. Rev. D 79, 094014 (2009).

[5] I. G. Alekseev et al.: Phys. Rev. D 79, 094014 (2009).

[6] S. R. Klein, J. Nystrand, Phys. Rev. C 60, 014903 (1999), STARLIGHT webpage, http://starlight.hepforge.org/

[7] J. D. Jackson,“ Classical Electrodynamics”, John Wiley \& Sons, Inc. (1999).

[8] A. Mucke, R. Engel, J.P. Rachen, R.J. Protheroe, and T. Stanev, Comput. Phys. Commun. 123, 290-314 (2000); SOPHIA webpage, http://homepage.uibk.ac.at/ c705282/SOPHIA.html

[9] G. Mitsuka, Eur. Phys. J. C 75, 614 (2015).

[10] O. Eyser, PSTP workshop, Bochum, Sept. (2015).

[11] D. C. Carey et al.(FNAL-E704 Collaboration), Phys. Rev. Lett. 64, 357 (1990).

[12] Elliot Leader, "Spin in Particle Physics", Cambridge University Press (2001). 